ARTICLE

Received 20 Jun 2016 | Accepted 5 Jan 2017 | Published 27 Feb $2017 \quad$ DOl: 10.1038/ncomms14549 $\quad$ OPEN

\title{
Superconducting parity effect across the Anderson limit
}

Sergio Vlaic ${ }^{1}$, Stéphane Pons ${ }^{1}$, Tianzhen Zhang ${ }^{1}$, Alexandre Assouline ${ }^{1}$, Alexandre Zimmers ${ }^{1}$, Christophe David ${ }^{2}$, Guillemin Rodary ${ }^{2}$, Jean-Christophe Girard ${ }^{2}$, Dimitri Roditchev ${ }^{1} \&$ Hervé Aubin ${ }^{1}$

How small can superconductors be? For isolated nanoparticles subject to quantum size effects, P.W. Anderson in 1959 conjectured that superconductivity could only exist when the electronic level spacing $\delta$ is smaller than the superconducting gap energy $\Delta$. Here we report a scanning tunnelling spectroscopy study of superconducting lead $(\mathrm{Pb})$ nanocrystals grown on the (110) surface of InAs. We find that for nanocrystals of lateral size smaller than the Fermi wavelength of the $2 \mathrm{D}$ electron gas at the surface of $\ln A s$, the electronic transmission of the interface is weak; this leads to Coulomb blockade and enables the extraction of electron addition energy of the nanocrystals. For large nanocrystals, the addition energy displays superconducting parity effect, a direct consequence of Cooper pairing. Studying this parity effect as a function of nanocrystal volume, we find the suppression of Cooper pairing when the mean electronic level spacing overcomes the superconducting gap energy, thus demonstrating unambiguously the validity of the Anderson criterion.

1'LPEM, ESPCI Paris, PSL Research University, CNRS, Sorbonne Universités, UPMC University of Paris 6, 10 rue Vauquelin, Paris F-75005, France. ${ }^{2}$ Centre de Nanosciences et de Nanotechnologies, CNRS, Univ. Paris-Sud, Universités Paris-Saclay, C2N-Marcoussis, Marcoussis 91460, France. Correspondence and requests for materials should be addressed to H.A. (email: herve.aubin@espci.fr). 
- he addition energy of an electron to a superconducting island, weakly coupled to the environment by the capacitance $C_{\Sigma}$, is given by (see Methods):

$$
E_{\text {even (odd) }}=\frac{e^{2}}{C_{\Sigma}}+(-) 2 \Delta+\delta
$$

where the first term is the Coulomb energy, the second term depends on the parity of electron occupation number as a consequence of the formation of a Cooper pair ${ }^{1,2}$ and the third term is the electronic level spacing in the island. This parity effect has been observed in large: $1 \mu \mathrm{m}$ micro-fabricated $\mathrm{Al}$ islands, through direct measurement of the charge capacitance of the island ${ }^{2}$, through the even-odd modulation of the addition energy in single-electron transistors ${ }^{3-6}$ or the parity dependence of the Josephson current in Cooper pair transistors ${ }^{7-9}$.

Until now, the parity effect on the addition energy has never been observed in small nanocrystals (NCs) near the Anderson limit ${ }^{10}$, reached at a volume about $V_{\text {Anderson }} \simeq 100 \mathrm{~nm}^{3}$, where the mean electronic level spacing $\langle\delta\rangle$ equals the superconducting gap energy $\Delta$.

In single-electron transistors fabricated with nanosized superconducting grains of aluminium ${ }^{11,12}$, the $2 e$ modulation of the addition energy could not be observed directly. Also, because only a few devices could be fabricated, testing the Anderson criterion was not possible with this approach. Indirect indications for the disappearance of superconductivity in small superconducting grains came from magnetization measurements ${ }^{13,14}$; because these measurements were averaged over macroscopic quantities of NCs, the link to the Anderson limit remained ambiguous.

In this work, we present a new system that enables a study of single and isolated NCs across the Anderson limit, where the NCs can be reproducibly obtained in large quantities. The superconducting gap energy and the transition temperature are measured through a study of the superconducting parity effect in the addition energy of the NCs. This constitutes an alternative approach to conventional tunnelling measurement of the superconducting gap in the quasiparticle excitation spectrum, which cannot be accessed as a consequence of the Coulomb gap at zero bias.

\section{Results}

Sample preparation. The observation of the parity effect is challenging as it requires clean systems, free of impurity states responsible for the so-called quasiparticle poisoning 9,15 . Furthermore, scanning tunnelling spectroscopy of isolated NCs requires, in addition to the tip-NC tunnel barrier, a second tunnel barrier between the NC and the conducting substrate ${ }^{16,17}$, as sketched in Supplementary Fig. 1.

In this work, the $\mathrm{Pb} \mathrm{NCs}$ are obtained by thermal evaporation of a nominal 0.3 monolayer of $\mathrm{Pb}$ on the (110) surface of InAs heated at $T=150^{\circ} \mathrm{C}$. The (110) surface is obtained by cleaving an n-type InAs substrate in ultra-high vacuum at a base pressure $P \sim 10^{-10}$ mbar. Two distinct samples (A and $B$ ) have been prepared with slightly different NC concentrations and sizes. The volume of the NCs ranges from $20 \mathrm{~nm}^{3} \simeq 0.2 V_{\text {Anderson }}$ to
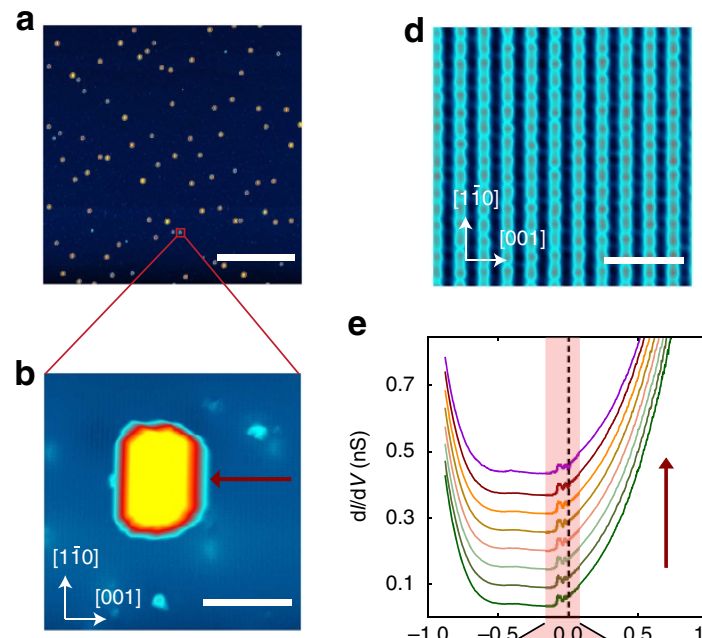

e
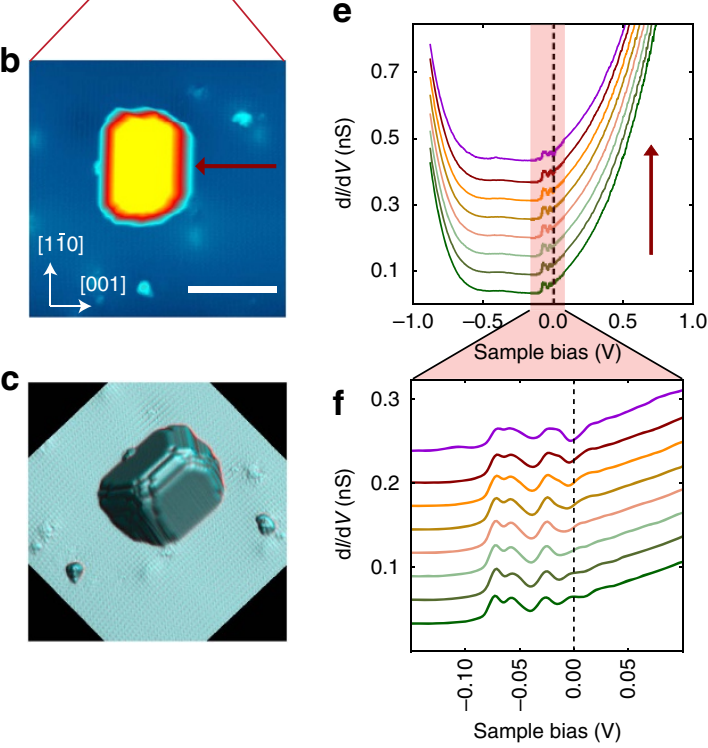

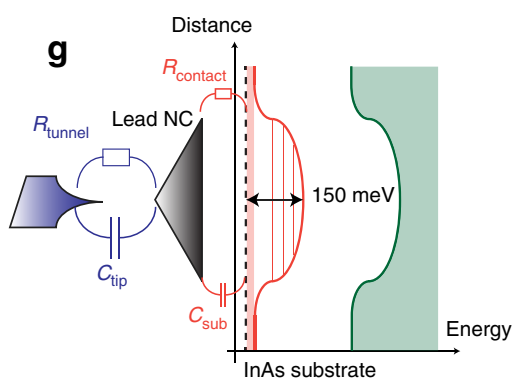

h

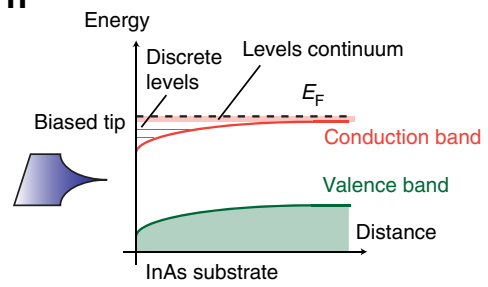

Figure 1 | Pb NCs on InAs (110). (a) $1 \mu \mathrm{m} \times 1 \mu \mathrm{m}$ topographic STM image (1V, $30 \mathrm{pA})$ of Pb NCs grown on the (110) InAs surface of sample A. Scale bar, $300 \mathrm{~nm}$. (b) Zoom on $30 \mathrm{~nm} \times 30 \mathrm{~nm}$ area, showing a Pb NC. Scale bar, $10 \mathrm{~nm}$. (c) 3D Laplacian $\Delta_{x y} z(x, y)$ image of a NC. (d) $6.5 \mathrm{~nm} \times 6.5 \mathrm{~nm}$ atomic resolution image of $\ln A s(110)$ obtained near the NC. Scale bar, $2 \mathrm{~nm}$. (e) DC measured at several distances from the Pb NC along the red arrow in b. (f) Zoom at low bias showing the conductance peaks due the discrete levels of the tip-induced quantum dot. (g) Sketch of the band bending below the Pb NC due to the pinning of the Fermi level at the charge neutrality level. (h) Sketch of the band bending induced by the tip leading to the formation of a quantum dot. 


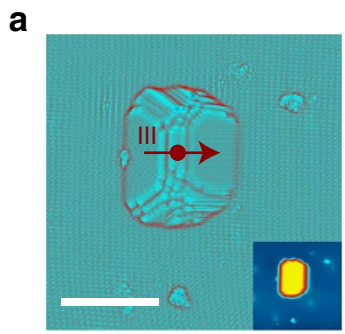

b

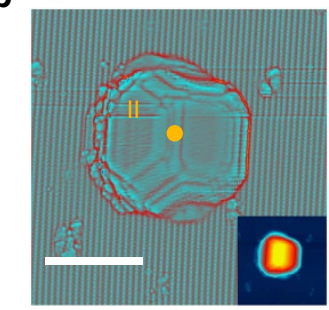

C

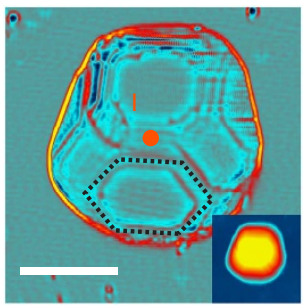

i

d
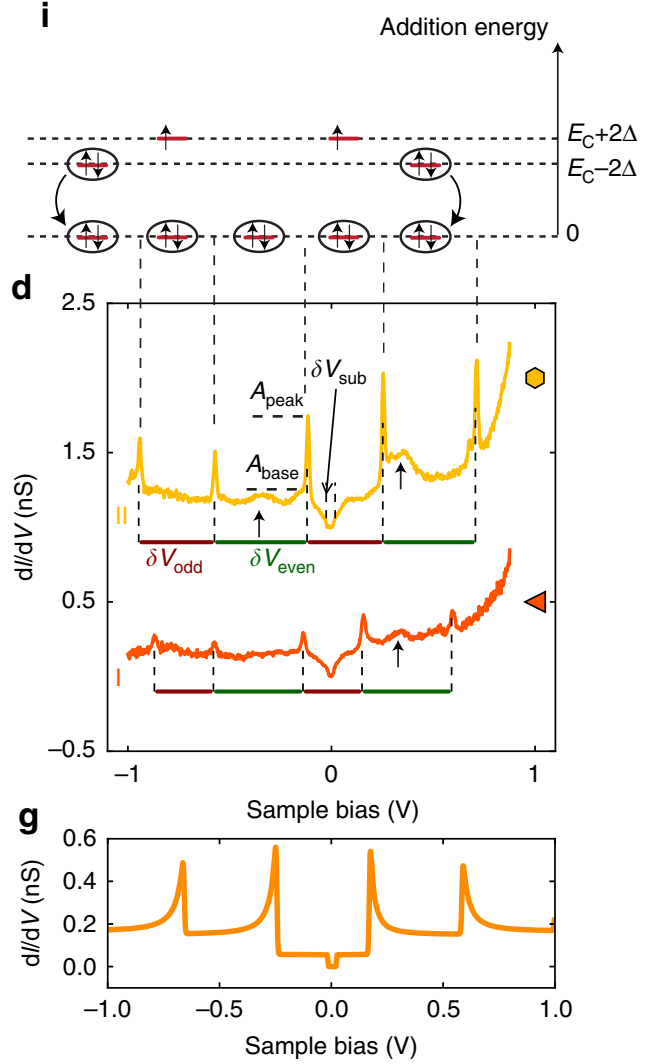

e

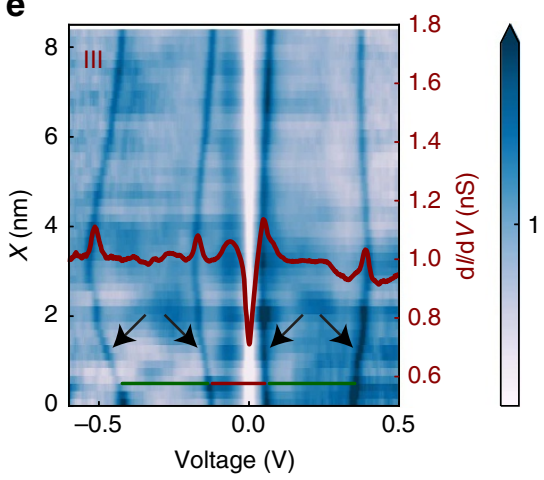

f

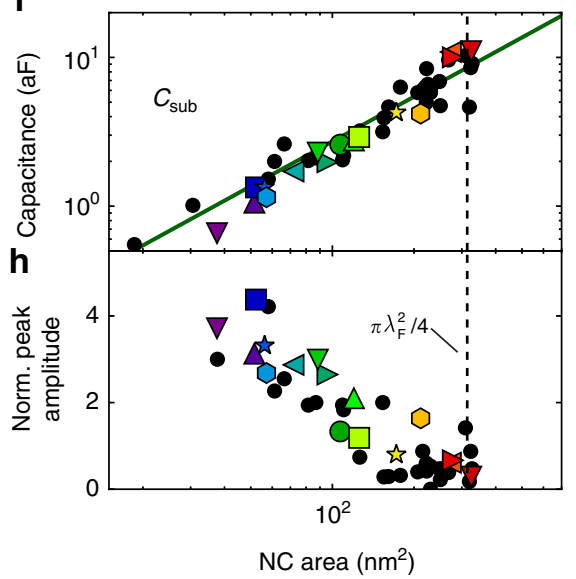

Figure 2 | Pb NCs in the regime of Coulomb blockade. (a-c) $30 \mathrm{~nm} \times 30 \mathrm{~nm}$ Laplacian $\Delta_{x y} z(x, y)$ topographic images (30 pA, $\left.1 \mathrm{~V}\right)$ of NCs of decreasing size, labelled I to III, where the hexagonal shape of the (111) facets is visible, as shown by the dash line in $\mathbf{c}$. The scale bars correspond to $10 \mathrm{~nm}$. The insets show the corresponding topographic STM images. (d) DC measured at the centre of NC I and II, indicated by dots in $\mathbf{b}, \mathbf{c}$. The addition voltages $\delta V_{\text {odd }}$ and $\delta V_{\text {even }}$ can be identified for each curve. The black arrows indicate the local maxima in the density of states due to quantum well states. The coloured symbols identify the corresponding data points in $\mathbf{f}, \mathbf{h}$, and Fig. 5. (e) DC map as function of sample bias and distance measured on NC III along the red arrow shown in $\mathbf{a}$. The black arrows indicate the Coulomb peak lines. (f) Capacitance $C_{\text {sub }}$ extracted from the Coulomb gap at zero bias. It scales linearly with the NC area. (g) Simulation of the DC for NC II using the weak coupling model ${ }^{26}$. (h) Normalized Coulomb peak amplitude $A_{\text {norm }}=\left(A_{\text {peak }}{ }^{-} A_{\text {base }}\right) /$ $A_{\text {base, }}$ this value decreases at the approach of the area $\pi \lambda_{F}^{2} / 4$. (i) Sketch of electron occupation of NC II.

$800 \mathrm{~nm}^{3} \simeq 8 V_{\text {Anderson }}$ while the height ranges from 1 unit cell $(0.495 \mathrm{~nm})$ to $5.2 \mathrm{~nm}$, see Supplementary Fig. 2 and Supplementary Note 1 for details on NC volume determination. The scanning tunnelling microscopy (STM) topographic images (Fig. 1a-c; Supplementary Fig. 3) for sample A and sample B, respectively, show that $\mathrm{Pb}$ grows in the Volmer-Weber, that is, Island mode ${ }^{18}$. The three dimensional Laplacian image $\Delta_{x y} z(x, y)$ (Fig. 1c) shows that the NCs are well crystallized and expose mostly the (111) planes of the cubic face-centred $\mathrm{Pb}$ structure, as indicated by the observation of the characteristic hexagonal shape of the (111) facets. Surrounding these NCs, the surface remains free from adsorbate, as atomic resolution images of the (110) InAs surface prove (Fig. 1d).

Tip-induced QDot on the InAs surface. Figure 1e shows the differential conductance (DC) $\mathrm{d} I / \mathrm{d} V$ measured on the InAs surface at several distances, from 0 to $10 \mathrm{~nm}$, of a $\mathrm{Pb} \mathrm{NC}$. The data are measured at $T=1.3 \mathrm{~K}$, unless indicated otherwise, using a standard lock-in procedure (see Methods). The data indicate that the Fermi level is in the conduction band of InAs as expected for this $\mathrm{n}$-doped sample. With a sulphur dopant concentration, $\mathrm{ND} \sim 6 \times 10^{16} \mathrm{~cm}^{-3}$, the Fermi level is $21 \mathrm{meV}$ above the conduction band minimum. A zoom on these spectra (Fig. 1f) shows multiple peaks that result from the discrete levels of the tipinduced quantum dot (QDot), a phenomena that has also been observed in previous works ${ }^{19}$. This demonstrates that $\mathrm{Pb}$ deposition on InAs do not produce any significant defects and doping. Indeed, in the presence of defects or adsorbate, the surface of III-V semiconductors present interface states that pin the Fermi level at the charge neutrality level $\mathrm{e}^{20,21}$ (Fig. 1g). For InAs, this level is located $150 \mathrm{meV}$ above its conduction band minimum, which leads to the formation of an electron accumulation layer as shown by numerous photoemission experiments ${ }^{20,22}$. In contrast, perfectly clean (110) surfaces do not present any interface states and consequently the Fermi level is not pinned. Thus, the electric field from the STM tip can easily shift the conduction band and generates the so-called tip-induced $\mathrm{QDot}^{19}$, as sketched in Fig. 1h. While the energy of the QDot levels can shift on long distances, see Supplementary Fig. 4, as a consequence of variations in the electrostatic environment due to the random distribution of $\mathrm{Pb} \mathrm{NCs}$ and sulphur dopants, we see (Fig. 1f) that the QDdot levels are not altered on short distances $(<10 \mathrm{~nm})$ near the NCs. Only a weak broadening of the QDot levels is observed, likely a consequence of their weak tunnel coupling with the $\mathrm{Pb}$ NCs.

Coulomb blockade and nature of the tunnel barrier. On NCs of three distinct sizes shown in Fig. 2a-c, representative DC spectra are shown in Fig. 2d,e. They display a Coulomb gap at zero bias of width $\delta V_{\text {sub }}=e /\left(C_{\text {sub }}+C_{\text {tip }}\right)$, where $C_{\text {sub }}\left(C_{\text {tip }}\right)$ is the capacitance between the $\mathrm{NC}$ and the substrate (tip). The data also display sharp Coulomb peaks where the voltage interval between the 


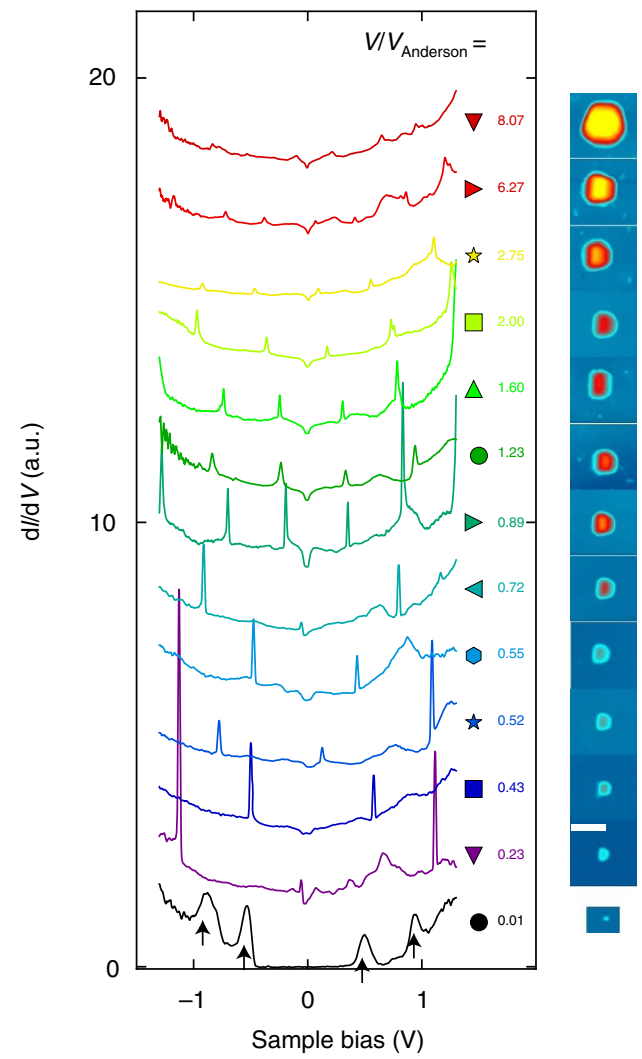

Figure 3 | DCs for increasing NC volume. The coloured symbols identify the corresponding data points in Figs 2f,h and 5. For each spectrum, the corresponding $\mathrm{NC}$ and the volume ratio $\mathrm{V} / \mathrm{V}_{\text {Anderson }}$ are shown on the right. Note that for the smallest NC (bottom black curve) no Coulomb peaks are observed, instead a large Coulomb gap and broad quantum well peaks are observed.

peaks provides the addition voltage $\delta V_{\text {add }}$ for an electron, which is related to the addition energy by: $\delta V_{\text {add }}=E_{\text {add }} / e \eta$, where $\eta=\frac{C_{\text {tip }}}{C_{\text {tip }}+C_{\text {sub }}}$ is the arm lever; see the Methods section for a derivation of these relations. Furthermore, the DCs may also display broad additional peaks, of weak amplitude in large NCs, $V / V_{\text {Anderson }}>1$, as indicated by arrows in Fig. 2d, but of large amplitude in small NCs, $V / V_{\text {Anderson }} \ll 1$, as indicated by arrows in Fig. 3. These broad peaks are the signature of quantum well states in the $\mathrm{Pb}$ NCs due to strong confinement in the $<111>$ direction as observed in scanning tunnelling studies of thin layers of $\mathrm{Pb}^{23}$.

The colour map in Fig. 2e shows that $\delta V_{\text {add }}$ changes slightly with the tip position above the $\mathrm{NC}$, as consequence of the variation in the tip-NC capacitance $C_{\text {tip. }}$. Figure 3 shows the DCs for 13 additional NCs, from which the capacitance $C_{\text {sub }}$ is extracted and shown as coloured symbols in Fig. $2 \mathrm{f}$ and Supplementary Fig. 5. On these last plots, data points shown as black circles of 24 other NCs are also included, for which the DCs are not shown. Figure $2 \mathrm{f}$ shows that $C_{\text {sub }}$ increases linearly with the area $A$ as $C_{\text {sub }}=A \varepsilon / d$, using $\varepsilon=12.3$, the dielectric constant of InAs and $d=4 \mathrm{~nm}$ for the effective tunnel barrier thickness.

As no dielectric insulator has been deposited on the surface and no Schottky barrier exists at metal-InAs interfaces ${ }^{21,22}$, the origin of the tunnel barrier and the meaning of the thickness $d$ appear clearly only after one realizes that the Fermi wavelength of the two-dimensional (2D) gas in InAs is larger than the lateral size of the NCs. At the interface between the Pb NC and InAs, the Fermi energy in InAs is at the charge neutrality level, $E_{\mathrm{F}}=150 \mathrm{meV}$ (refs 21,22), which gives for the Fermi wavelength $\lambda_{\mathrm{F}}=20 \mathrm{~nm}$. As known from numerous works with quantum point-contacts formed in $2 \mathrm{D}$ electron gas ${ }^{24,25}$, the transmission coefficient $T$ decreases for constrictions smaller than the Fermi wavelength. Because a NC covers only a fraction of the area $\simeq \lambda_{\mathrm{F}}^{2}$, its transmission coefficient with the $2 \mathrm{D}$ gas is significantly smaller than one, which explains the observation of the Coulomb blockade. For a small NC, the weak coupling model $^{26}$ can be used to describe the data, as shown in Fig. $2 \mathrm{~g}$. This model shows that the contact impedance is of the order of $R_{\text {contact }} \sim 10 \mathrm{M} \Omega$, implying that the transmission coefficient $T=R_{\text {contact }} e^{2} / h=0.0025$ is weak as anticipated. In this model, the magnitude of the Coulomb peaks increases with the ratio $R_{\text {tunnel }} / R_{\text {contact }}$, as observed on the DC curves measured as function of tip height (Supplementary Fig. 6). Figure $2 \mathrm{~h}$ shows the amplitude of the Coulomb peak, normalized to its base value, as function of NC area. The amplitude is constant for small area $\left(<100 \mathrm{~nm}^{2}\right)$ but decreases quickly for area approaching $\pi \lambda_{\mathrm{F}}^{2} / 4 \simeq 300 \mathrm{~nm}^{2}$. This behaviour cannot be described by the weak coupling model just discussed; however, it can be understood by considering models of Coulomb blockade in the strong coupling regime $\mathrm{e}^{27,28}$. These models show that the Coulomb oscillations disappear when $T$ approaches unity, when charge fluctuations between the NC and the substrate become significant. Figure 3 shows that the Coulomb peaks of the largest NCs have almost completely disappeared. The fact that the amplitude of the Coulomb peaks decreases for NCs area approaching $\lambda_{\mathrm{F}}^{2}$ confirms our interpretation that the tunnel barrier is due to a quantum constriction of the electronic wave function at the interface between the $\mathrm{NC}$ and the $2 \mathrm{D}$ gas. Thus, the dielectric thickness $d=4 \mathrm{~nm}$ extracted from $C_{\text {sub }}$ above is actually set by the Debye length of the $2 \mathrm{D}$ gas and $C_{\text {sub }}$ actually corresponds to the quantum capacitance of InAs.

Superconducting parity effect. Owing to this highly clean type of tunnel junction, free from quasiparticle poisoning, the superconducting parity effect in the NCs can be observed through the even-odd modulation of the addition voltage, as shown in Figs $2 \mathrm{~d}, \mathrm{e}, 3$ and 4 . The addition voltages can be precisely extracted due to the sharpness of the Coulomb peaks, which voltage positions are obtained through a fit with a Lorentz function (Supplementary Fig. 7). As sketched in Fig. 2i and shown by equation (1), the addition voltage $\delta V_{\text {even }}$ for injecting an electron in an even parity NC is higher than $\delta V_{\text {odd }}$ for injecting an electron in an odd parity $\mathrm{NC}$, where the energy difference is given by the binding energy of the Cooper pair. Figure 4a shows the DCs for a large $\mathrm{NC}, V / V_{\text {Anderson }}=1.6$, as function of temperature. The corresponding addition voltages, shown in Fig. $4 \mathrm{~b}$, are almost equal above $T_{\mathrm{c}}=7.2 \mathrm{~K}$, the superconducting transition temperature of bulk $\mathrm{Pb}$. However, an even-odd modulation is observed at low temperature $T=1.3 \mathrm{~K}$. The difference in the addition energies between two successive charge configurations is obtained from $\delta E=e \eta\left(\delta V_{\text {even }}-\delta V_{\text {odd }}\right)$. For this large NC, four Coulomb peaks are observed, which provide three distinct addition voltages indicated by the horizontal bars. From these addition voltages, two distinct values of the addition energy difference $\delta E$ between two charge configurations are obtained and given by $\delta E=\eta\left(\delta V_{\text {Head }}-\delta V_{\text {Tail }}\right)$, where the head (tail) refers to the coloured arrows in the panel. These two values of $\delta E$ are shown in Fig. $4 \mathrm{c}$ as the function of temperature. Their values are near zero at high temperature, $\delta E_{\mathrm{HT}} \sim 0$, and increase below $T_{\mathrm{c}}=7.2 \mathrm{~K}$ to reach, at low temperature, the theoretically expected value $\left|\delta E_{\mathrm{LT}}\right| \sim 4 \Delta_{\text {bulk }}$ (ref. 1 ), where $\Delta_{\text {bulk }}=1.29 \mathrm{meV}$ is the superconducting gap of bulk $\mathrm{Pb}$. The value $\delta E_{\mathrm{LT}}$ changes sign as one goes from the difference between two addition energies 
a

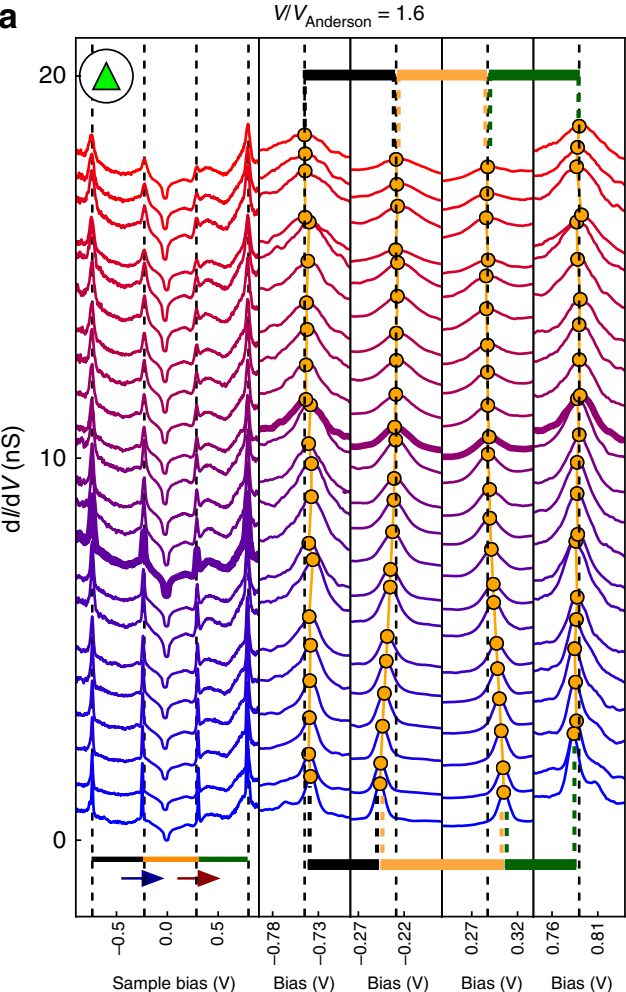

b

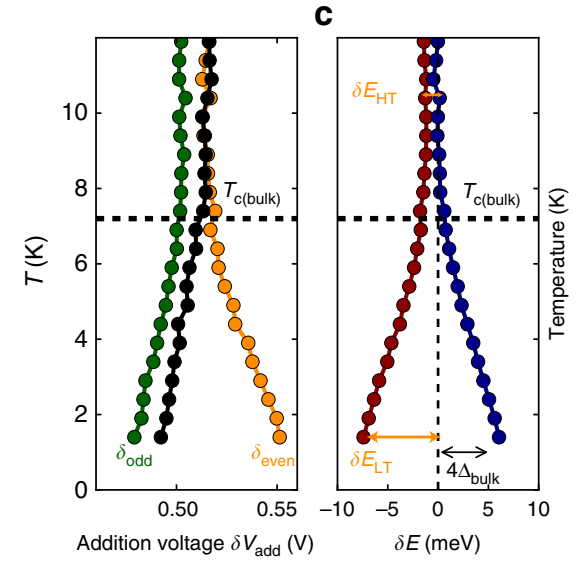

d

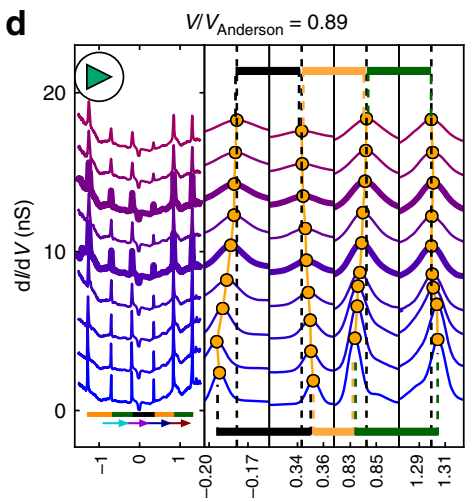

Sample bias (V) Bias (V) Bias (V) Bias (V) Bias (V)

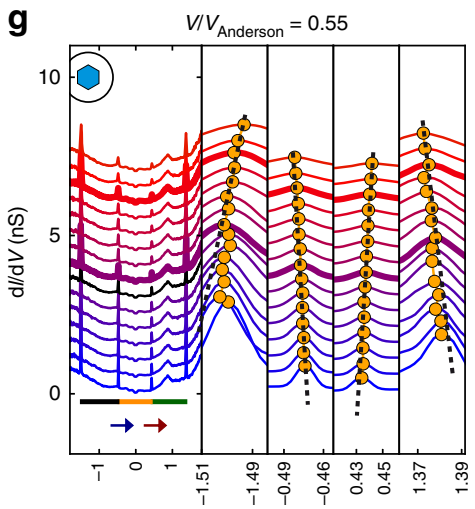

Sample bias (V) Bias (V) Bias (V) Bias (V) Bias (V)

j

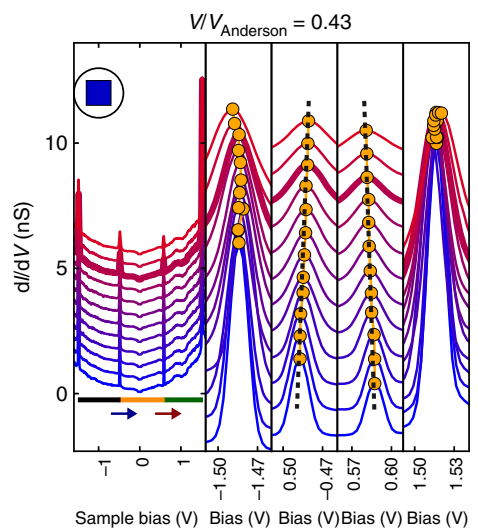

e

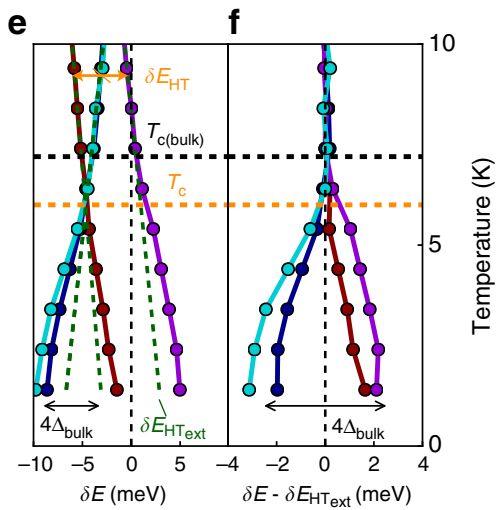

h

i

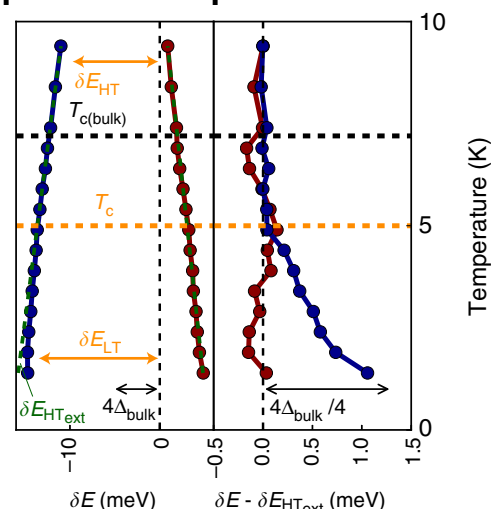

$\mathbf{k}$

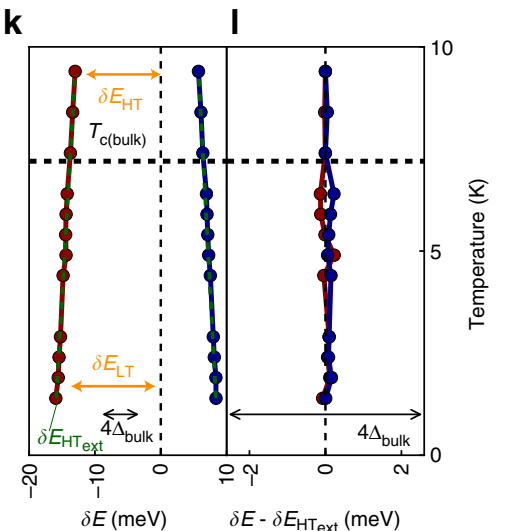

Figure 4 | Parity effect as function of temperature. DC and addition energies for four NCs of decreasing volume, where $V / V_{\text {Anderson }}$ is indicated on top of the panels. ( $\mathbf{a}, \mathbf{d}, \mathbf{g}, \mathbf{j})$ DC curves as function of temperature. The voltage separation between the Coulomb peaks, that is, the addition voltage, is indicated by the horizontal bars of different colours. In the same panels, zoom on the Coulomb peaks are shown where the maxima are indicated by orange dots. For $\mathbf{a}$, the addition voltages are plotted as the function of temperature in $\mathbf{b}$ with corresponding colours. The coloured symbols (top left of panels) identify the corresponding data points in Figs $2 f, h$ and 5 . ( $(\mathbf{c}, \mathbf{e}, \mathbf{h}, \mathbf{k})$ Difference in addition energies between two charge configurations given by $\delta E=\eta\left(\delta V_{\text {Head }}-\delta V_{\text {Tail }}\right)$, where the head (tail) refers to the arrows shown in the corresponding panels. (f,i,l) Difference $\delta E-\delta E_{\mathrm{HT}_{\text {ext }}}$, where the dash green line $\delta E_{\mathrm{HT}_{\mathrm{ext}}}$, is obtained from the extrapolation of $\delta E$ at high temperature. For $\mathbf{b}, \mathbf{c}, \mathbf{e}, \mathbf{f}, \mathbf{h}, \mathbf{i}, \mathbf{k}, \mathbf{l}$, the value $T_{\mathrm{c}}$ (bulk) is indicated as a black dash line. The extracted $T_{\mathrm{c}}$ is shown as orange dash lines. A double-headed arrow provides the scale for the energy gap $4 \Delta_{\text {bulk }}$ of bulk $\mathrm{Pb}$.

$\delta E=e \eta\left(\delta V_{\text {even }}-\delta V_{\text {odd }}\right)$ to the next difference $\delta E=e \eta\left(\delta V_{\text {odd }^{-}}\right.$ $\left.\delta V_{\text {even }}\right)$.

For NCs smaller than the Anderson volume (Fig. 4d,g,j), we observe that $\delta E_{\mathrm{HT}}$ is non-zero, which indicates that the electronic level spacing $\delta$ has now a significant contribution to the addition energy, following equation (1). The values of $\delta E_{\mathrm{HT}}$ are distinct between successive charge configurations. Indeed, in metallic systems, the electronic levels are randomly distributed as described by random matrix theory $(\mathrm{RMT})^{29}$. Collecting the values $\delta E_{\mathrm{HT}}$ for all NCs, Fig. 5a shows that, in average, the evolution of $\delta E_{\mathrm{HT}}$ with NC volume can be properly described by the relation:

$$
<\delta>=\frac{2(\pi \hbar)^{2}}{m^{*} k_{\mathrm{F} 1(2)} \text { Volume }}
$$

using $m^{*}=1.2 m_{\mathrm{e}}$ for the effective mass, where $k_{\mathrm{F} 1}=7.01 \mathrm{~nm}^{-1}$ and $k_{\mathrm{F} 2}=11.21 \mathrm{~nm}^{-1}$ are characteristic wavevectors of the two Fermi surfaces FS1 and FS2 of $\mathrm{Pb}$. 
a

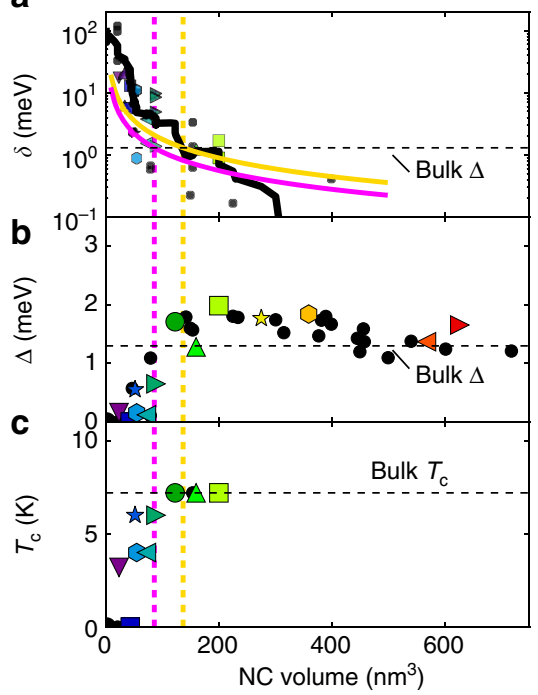

d

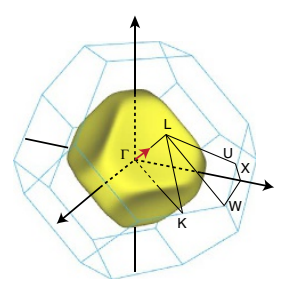

e

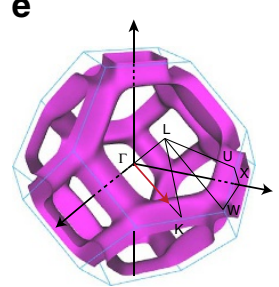

Figure 5 | Across the Anderson limit. (a) Level spacing extracted from the addition energies measured above $T_{\mathrm{c}}$. The experimental data (symbols) are highly scattered as a consequence of the random electronic level distribution. However, the average level spacing, shown by the smoothed black line, is of the order of magnitude of the calculated theoretical values shown as coloured lines. The horizontal dash line indicates the bulk superconducting energy gap. (b) Superconducting gap $\Delta$ extracted from the difference in addition energies between high and low temperature. The horizontal dash line indicates the bulk superconducting energy gap. (c) Transition temperature as the function of NC volume. The horizontal dash line indicates the bulk transition temperature $T_{\mathrm{c}}=7.2 \mathrm{~K}$. For all panels, the two vertical dash lines indicate the volumes where the level spacing reaches the superconducting energy gap at the wavevectors shown by red arrows on the two Fermi surfaces on the right. The coloured symbols identify the corresponding DC curves in the other figures. For the black circles, the DCs are not shown. (d) Fermi surface (FS1) of the hole-type band of $\mathrm{Pb}$. (e) Fermi surface (FS2) of the electron-type band of $\mathrm{Pb}$.

For the $\mathrm{NC}$ of volume $V / V_{\text {Anderson }}=0.89$ (Fig. $4 \mathrm{~d}$ ), while the level spacing $\delta E_{\mathrm{HT}}$ is large, the shift of the Coulomb peaks due to the parity effect is still dominating the temperature dependence and can be observed directly on the raw data and the addition energy difference $\delta E$ plotted as the function of temperature in Fig. 4e. A line $\delta E_{\mathrm{HT}_{\mathrm{ext}}}$ is extrapolated from high temperature and the difference $\delta E(T)-\delta E_{\mathrm{HT}_{\mathrm{ext}}}(T)$ gives the temperature dependence of the superconducting gap (Fig. 4f), which shows that the critical temperature $T_{\mathrm{c}} \simeq 6 \mathrm{~K}$ is smaller than the bulk value. The amplitude of the superconducting gap is obtained from $\Delta=\left(\delta E(T=1.2 \mathrm{~K})-\delta E_{\mathrm{HT}_{\mathrm{ext}}}(T=1.2 \mathrm{~K})\right) / 4$. For this $\mathrm{NC}$, the superconducting energy gap is about two times smaller than the bulk value, $\Delta=\Delta_{\text {bulk }} / 2$.

For the smaller $\mathrm{NC}$ of volume $V / V_{\text {Anderson }}=0.55$ (Fig. $4 \mathrm{~g}$ ), the level spacing $\delta E_{\mathrm{HT}}$ is larger and has a temperature dependence that dominates the shift of the Coulomb peaks with temperature. This shift could be the consequence of thermally induced electrochemical shifts or temperature-dependent strain or electric field effects. While the parity effect is barely visible on the raw data, using the procedure employed for the previous $\mathrm{NC}$, the temperature $T_{\mathrm{c}} \simeq 5 \mathrm{~K}$ value and the energy gap $\Delta \simeq \Delta_{\text {bulk }} / 4$ can be extracted (Fig. $4 \mathrm{i}$ ).

Finally, for the smallest NCs $V / V_{\text {Anderson }}=0.43(0.34)$, shown, respectively, in Fig. $4 \mathrm{j}$ and Supplementary Fig. $8 \mathrm{~h}$, they have the largest level spacing $\delta E_{\mathrm{HT}}$ and, even though the addition energies are measured with much higher resolution than the superconducting gap energy, no parity effect can be observed in Fig. 41 and Supplementary Fig. 8j, respectively.

For 13 NCs where the DCs have been acquired as the function of temperature, some of which are shown in Fig. 4 and Supplementary Fig. 8, the level spacing, the superconducting gap energy and the transition temperature are extracted and plotted (Fig. 5a-c), respectively. Upon reducing the NC volume, both quantities display a sharp decrease to zero when the level spacing becomes of the order of the superconducting gap energy, $\simeq 1 \mathrm{meV}$. See Supplementary Note 2 for a comparison with the results of Bose et al. ${ }^{17}$ on a system where the superconducting nanoparticles are strongly coupled to the normal substrate.

\section{Discussion}

Figure 5 suggests that superconductivity disappears when the mean level spacing at the Fermi surface of the electron-type band (Fig. 5e), increases up to the superconducting gap energy. This is consistent with recent theoretical calculations ${ }^{30}$ and STM measurements ${ }^{31}$, which have shown that electron-phonon coupling is stronger for this electron-type band owing to its $\mathrm{p}-\mathrm{d}$ character. Regarding the Bardeen-Cooper-Schrieffer ratio $k_{\mathrm{B}} T_{\mathrm{c}} / \Delta$, within the experimental resolution, no significant deviation from the bulk value has been observed.

To summarize, we have found that a $2 \mathrm{D}$ electron gas of large Fermi wavelength constitutes an ideal substrate for studying Coulomb blockade in nanosized NCs evaporated in an ultrahigh vacuum environment. This discovery leads us to observe, for the first time by STM, the parity effect and quantum confinement in isolated superconducting NCs and enabled the first demonstration of the Anderson criterion for the existence of superconductivity at single NC level. Furthermore, this new insight on the superconductor-InAs interface is of interest for topological superconductivity where Majorana islands are generated by depositing a superconductor on InAs nanowires ${ }^{32,33}$.

\section{Methods}

Relation between sample bias and energies. The Coulomb gap at zero bias results from Coulomb blockade that prevent charge fluctuations in the NC. As sketched in Supplementary Fig. 1, Coulomb blockade is lifted when the Fermi level of either one of the electrodes is aligned with one of the excited levels of the NC. Thus the amplitude of the Coulomb gap observed in the DC is given by

$\delta V_{\text {sub }}=\frac{e}{C_{\Sigma}}=2 \times \frac{E_{\mathrm{C}}}{e}$, with $E_{C}=\frac{e^{2}}{2 C_{\Sigma}}$.

The Coulomb peaks observed at higher voltages result from the shift of the electrochemical potential of the NC upon increasing the voltage bias across the double junction. This shift is given by:

$$
\frac{\Delta \mu}{e}=\eta V_{\text {Bias }}
$$

with:

$$
\eta=\frac{C_{\text {tip }}}{C_{\text {tip }}+C_{\text {sub }}}
$$

Charge states with increased number of electrons become accessible when the electrochemical potential changes by $2 \times E_{\mathrm{C}}$. Thus, the voltage difference between two charge states is given by:

$$
\Delta V_{\text {add }}=\frac{1}{\eta} \times \frac{2 E_{\mathrm{C}}}{e}=\frac{e}{C_{\text {tip }}}
$$

This formula shows that the addition voltage depends only on the capacitance $C_{\text {tip }}$ and not on the capacitance $C_{\text {sub }}$, as shown in Supplementary Fig. 1b, where a simulation of the conduction spectrum, using the Hanna and Tinkham model ${ }^{26}$ for two distinct values of the capacitance $C_{\text {sub }}$.

Addition energies. Following refs 1,34 , the total energy of a NC with $N$ electrons is given by:

$$
\begin{aligned}
& E(N)=\frac{(N e)^{2}}{2 C_{\Sigma}}+E_{0}(N) \\
& E_{0}(N)=\left\{\begin{array}{l}
\Delta \text { for odd } N, \\
0 \text { for even } N,
\end{array}\right.
\end{aligned}
$$


The electrochemical potential of a NC with an even (odd) $N(N+1)$ number of electrons is given by:

$$
\begin{aligned}
& \mu(N)=E(N+1)-E(N)=\left(N+\frac{1}{2}\right) \frac{e^{2}}{C_{\Sigma}}+\Delta \\
& \mu(N+1)=E(N+2)-E(N+1)=\left(N+\frac{3}{2}\right) \frac{e^{2}}{C_{\Sigma}}-\Delta
\end{aligned}
$$

From these last equations, one obtains the addition energies for a NC with an even (odd) $N(N+1)$ number of electrons:

$$
\begin{aligned}
& E_{\text {even }}=\mu(N)-\mu(N-1)=\frac{e^{2}}{C_{\Sigma}}+2 \Delta \\
& E_{\text {odd }}=\mu(N+1)-\mu(N)=\frac{e^{2}}{C_{\Sigma}}-2 \Delta
\end{aligned}
$$

Thus, the difference of addition energies between two successive charge states is given by:

$$
\delta E=E_{\text {even }}-E_{\text {odd }}=4 \Delta
$$

When the electronic spectrum of the NC is discrete, the level spacing $\delta$ should be included in the addition energy.

$$
E_{\text {even (odd) }}=\frac{e^{2}}{C_{\Sigma}}+(-) 2 \Delta+\delta
$$

Random level distribution. In metallic NCs, the electronic level distribution is described by $\mathrm{RMT}^{29,35}$. In a NC with strong spin-orbit coupling, RMT predicts that the level spacing should be described by a Gaussian symplectic ensemble. For this level distribution, shown in Supplementary Fig. 9, the width of the distribution, that is, the s.d., is equal to $\sigma \simeq\langle\delta>$ (refs 29,36). Between two successive charge states, the addition energy can fluctuate by an amount of the order of $\sigma$,

consequently, in average, the difference in addition energies between two successive charge states is given by:

$$
\delta E_{\mathrm{LT}}=E_{\text {even }}-E_{\text {odd }}=4 \Delta+<\delta>
$$

At temperatures above the superconducting transition temperature:

$$
\delta E_{\mathrm{HT}}=<\delta>
$$

Thus, an estimation of the level spacing can be obtained by a measure of the difference in the addition energies above $T_{\mathrm{C}}$.

Furthermore, the gap amplitude can be obtained from:

$$
\Delta=\left(\delta E_{\mathrm{LT}}-\delta E_{\mathrm{HT}}\right) / 4
$$

Measurements details. The microscope used is a low temperature, $T_{\text {base }}=1.3 \mathrm{~K}$, Joule-Thomson STM from SPECS accommodated with a preparation chamber operating in ultra-high vacuum at a base pressure $P \sim 10^{-10}$ mbar. The DC curves $\mathrm{d} I / \mathrm{d} V$ are measured with a standard lock-in procedure. An a.c. signal of amplitude $\simeq 1 \mathrm{meV}$ and frequency $\sim 777 \mathrm{~Hz}$ is employed.

Data avaibility. The data that support the main findings of this study are available from the corresponding author upon request.

\section{References}

1. Averin, D. V. \& Nazarov, Y. V. Single-electron charging of a superconducting island. Phys. Rev. Lett. 69, 1993-1996 (1992).

2. Lafarge, P., Joyez, P., Esteve, D., Urbina, C. \& Devoret, M. H. Two-electron quantization of the charge on a superconductor. Nature 365, 422-424 (1993).

3. Tuominen, M. T., Hergenrother, J. M., Tighe, T. S. \& Tinkham, M. Experimental evidence for parity-based 2e periodicity in a superconducting single-electron tunneling transistor. Phys. Rev. Lett. 69, 1997-2000 (1992).

4. Eiles, T., Martinis, J. \& Devoret, M. Even-odd asymmetry of a superconductor revealed by the Coulomb blockade of Andreev reflection. Phys. Rev. Lett. 70, 1862-1865 (1993).

5. Lafarge, P., Joyez, P., Esteve, D., Urbina, C. \& Devoret, M. H. Measurement of the even-odd free-energy difference of an isolated superconductor. Phys. Rev. Lett. 70, 994-997 (1993).

6. Higginbotham, A. P. et al. Parity lifetime of bound states in a proximitized semiconductor nanowire. Nat. Phys. 11, 1017-1021 (2015).

7. Joyez, P., Lafarge, P., Filipe, A., Esteve, D. \& Devoret, M. H. Observation of parity-induced suppression of Josephson tunneling in the superconducting single electron transistor. Phys. Rev. Lett. 72, 2458-2461 (1994).

8. Aumentado, J., Keller, M. W., Martinis, J. M. \& Devoret, M. H. Nonequilibrium quasiparticles and 2 e periodicity in single-cooper-pair transistors. Phys. Rev. Lett. 92, 066802 (2004).

9. van Woerkom, D. J., Geresdi, A. \& Kouwenhoven, L. P. One minute parity lifetime of a NbTiN Cooper-pair transistor. Nat. Phys. 11, 547-550 (2015).

10. Anderson, P. Theory of dirty superconductors. J. Phys. Chem. Solids 11, 26-30 (1959).
11. Ralph, D. C., Black, C. T. \& Tinkham, M. Spectroscopic measurements of discrete electronic states in single metal particles. Phys. Rev. Lett. 74, 3241-3244 (1995).

12. von Delft, J. \& Ralph, D. Spectroscopy of discrete energy levels in ultrasmall metallic grains. Phys. Rep. 345, 61-173 (2001).

13. Reich, S., Leitus, G., Popovitz-Biro, R. \& Schechter, M. Magnetization of small lead particles. Phys. Rev. Lett. 91, 147001 (2003).

14. Zolotavin, P. \& Guyot-Sionnest, P. Meissner effect in colloidal $\mathrm{Pb}$ nanoparticles. ACS Nano 4, 5599-5608 (2010).

15. Savin, A. M. et al. Parity effect in $\mathrm{Al}$ and $\mathrm{Nb}$ single electron transistors in a tunable environment. Appl. Phys. Lett. 91, 063512 (2007).

16. Hong, I.-P., Brun, C., Pivetta, M., Patthey, F. \& Schneider, W.-D. Coulomb blockade phenomena observed in supported metallic nanoislands. Front. Phys. 1, 1-8 (2013).

17. Bose, S. et al. Observation of shell effects in superconducting nanoparticles of Sn. Nat. Mater. 9, 550-554 (2010).

18. Brune, H. Microscopic view of epitaxial metal growth: nucleation and aggregation. Surf. Sci. Rep. 31, 125-229 (1998).

19. Dombrowski, R., Steinebach, C., Wittneven, C., Morgenstern, M. \& Wiesendanger, R. Tip-induced band bending by scanning tunneling spectroscopy of the states of the tip-induced quantum dot on InAs(110). Phys. Rev. B 59, 8043-8048 (1999).

20. Tersoff, J. Theory of semiconductor heterojunctions: the role of quantum dipoles. Phys. Rev. B 30, 4874-4877 (1984)

21. Mönch, W. Semiconductor Surfaces and Interfaces (Springer, 2001).

22. Morgenstern, M. et al. Scanning tunneling microscopy of two-dimensional semiconductors: spin properties and disorder. Physica E 44, 1795-1814 (2012).

23. $\mathrm{Su}, \mathrm{W}$. B. et al. Correlation between quantized electronic states and oscillatory thickness relaxations of $2 \mathrm{D} \mathrm{Pb}$ Islands on $\mathrm{Si}(111)-(7 \tilde{\mathrm{A}}-7)$ surfaces. Phys. Rev. Lett. 86, 5116-5119 (2001).

24. van Wees, B. J. et al. Quantized conductance of point contacts in a twodimensional electron gas. Phys. Rev. Lett. 60, 848-850 (1988).

25. Pasquier, C. et al. Quantum limitation on Coulomb blockade observed in a 2D electron system. Phys. Rev. Lett. 70, 69-72 (1993).

26. Hanna, A. E. \& Tinkham, M. Variation of the Coulomb staircase in a twojunction system by fractional electron charge. Phys. Rev. B 44, 5919-5922 (1991).

27. Matveev, K. A. Coulomb blockade at almost perfect transmission. Phys. Rev. B 51, 1743-1751 (1995).

28. Aleiner, I., Brouwer, P. \& Glazman, L. Quantum effects in Coulomb blockade. Phys. Rep. 358, 309-440 (2002).

29. Alhassid, Y. The statistical theory of quantum dots. Rev. Mod. Phys. 72, 895-968 (2000).

30. Floris, A., Sanna, A., Massidda, S. \& Gross, E. K. U. Two-band superconductivity in $\mathrm{Pb}$ from ab initio calculations. Phys. Rev. B 75, 1-6 (2007).

31. Ruby, M., Heinrich, B. W., Pascual, J. I. \& Franke, K. J. Experimental demonstration of a two-band superconducting state for lead using scanning tunneling spectroscopy. Phys. Rev. Lett. 114, 157001 (2015).

32. Das, A. et al. Zero-bias peaks and splitting in an AlInAs nanowire topological superconductor as a signature of Majorana fermions. Nat. Phys. 8, 887-895 (2012).

33. Albrecht, S. M. et al. Exponential protection of zero modes in Majorana Islands. Nature 531, 206-209 (2015).

34. Kouwenhoven, L. P., Austing, D. G. \& Tarucha, S. Few-electron quantum dots. Rep. Prog. Phys. 64, 701-736 (2001).

35. Halperin, W. Quantum size effects in metal particles. Rev. Modern Phys. 58, 533-606 (1986).

36. Mehta, M. L. Random Matrices (Academic Press, 2004).

\section{Acknowledgements}

We acknowledge fruitful discussions with M. Aprili, C. Delerue and B. Grandidier. H.A. and A.Z. acknowledge support from ANR grant 'QUANTICON' 10-0409-01, ANR grant 'CAMELEON’ 09-BLAN-0388-01, China Scholarship Council and labex Matisse. D.R. and S.P. acknowledge C'NANO Ile-de-France, DIM NanoK, for the support of the Nanospecs project.

\section{Author contributions}

H.A. proposed the experiment. C.D., G.R. and J.-C.G. designed the STM holder and prepared the InAs substrates. S.V., S.P., T.Z. and H.A. evaporated the lead NCs on the cleaved InAs substrate and carried out the STM measurements with the help of D.R. H.A. analysed the data and wrote the manuscript with the help of all authors.

\section{Additional information}

Supplementary Information accompanies this paper at http://www.nature.com/ naturecommunications 
Competing financial interests: The authors declare no competing financial interests.

Reprints and permission information is available online at http://npg.nature.com/ reprintsandpermissions/

How to cite this article: Vlaic, S. et al. Superconducting parity effect across the Anderson limit. Nat. Commun. 8, 14549 doi: 10.1038/ncomms14549 (2017).

Publisher's note: Springer Nature remains neutral with regard to jurisdictional claims in published maps and institutional affiliations. (c) (i) This work is licensed under a Creative Commons Attribution 4.0 International License. The images or other third party material in this article are included in the article's Creative Commons license, unless indicated otherwise in the credit line; if the material is not included under the Creative Commons license, users will need to obtain permission from the license holder to reproduce the material. To view a copy of this license, visit http://creativecommons.org/licenses/by/4.0/

(C) The Author(s) 2017 